\title{
From the bakery to the brain business: developing inducible yeast models of human neurodegenerative disorders
}

\author{
Alejandro Ocampo ${ }^{1}$ and Antoni Barrientos ${ }^{1,2}$ \\ 'The Department of Biochemistry and Molecular Biology, and 2 The Department \\ of Neurology, The John T. Macdonald Foundation Center for Medical Genetics, \\ University of Miami, Miller School of Medicine, Miami, FL, USA
}

BioTechniques 45:Pvii-Pxiv (October 2008)

doi $10.2144 / 000112746$

\begin{abstract}
In the last decade, the budding yeast Saccharomyces cerevisiae has been used as a model system to study the mechanisms of the human aging process and of age-associated neurodegenerative disorders such as Parkinson's, Huntington's, Alzheimer's, and amyotrophic lateral sclerosis. S. cerevisiae is a facultative aerobic, unicellular yeast, and despite their simplicity, yeast cells possess most of the same basic cellular machinery as neurons in the brain, including pathways required for protein homeostasis and energy metabolism. The power of yeast genetics and the use of high-throughput screening technologies have provided important clues concerning the pathophysiology of these disorders and the identification of candidate therapeutic targets and drugs. The yeast models are based on the expression of human disease proteins in yeast and recapitulate some of the cytotoxic features observed in patients. However, the currently available models mostly suffer from high-level protein expression that results in acute cytotoxicity, and from metabolic constraints when the models are based on extensively used, strong, galactose-inducible promoters. The models would increase their significance if they were based on continuous and tightly regulated gene expression systems for both activation and levels of expression. This would allow for more chronic cytotoxicity that better simulates the timing of events that occur during disease progression. Additionally, the use of metabolism-independent inducers would allow for the study of cell toxicities under conditions where the cells are forced to exclusively respire, thus more reliably modeling the highly oxidative neuronal metabolism. Here we have constructed yeast models of Huntington's disease based on the expression, under the control of different promoters, of the first exon of the huntingtin-containing polyglutamine tracts of both wild-type and mutant lengths. The different models are compared and evaluated.
\end{abstract}

\section{INTRODUCTION}

The unicellular yeast Saccharomyces cerevisiae has an extensive history of use in the areas of biotechnology and biomedicine. Known as baker's yeast or brewer's yeast, this organism has been used for centuries as leavening for bread and as a fermenter of alcoholic beverages (1). More recently, S. cerevisiae has been used as a valuable organism for studying the principles of microbiology, characterizing biochemical pathways, and understanding the biology of more complex eukaryotic organisms (2). Cellular activities conserved from yeast to humans include DNA replication, recombination, and repair; RNA transcription and translation; intracellular trafficking; enzymatic activities of general metabolism; and mitochondrial biogenesis (reviewed in Reference 3).

Yeast has certain properties that make it particularly suitable for biological studies, including rapid growth, the ability to be maintained in a haploid or diploid state, ease of mutant isolation, a well-defined genetic system, and a highly versatile DNA transformation system. Unlike many other microorganisms, S. cerevisiae is viable with numerous markers. Finally, being nonpathogenic, yeast can be handled with few precautions (3). S. cerevisiae was the first eukaryotic organism whose DNA sequence was completely known (4). Over the last 10 years, this knowledge fueled the use of this yeast as a model organism for studying the pathophysiology of human diseases. Recently, yeast was used to model the human aging process and complex neurodegenerative disorders, including amyotrophic lateral sclerosis (ALS), Parkinson's disease (PD), and Huntington's disease (HD) (reviewed in Reference 5). In humans, these neurodegenerative disorders are characterized by the progressive, selective loss of neurons in different areas of the brain associated with the misfolding of disease-specific proteins. Although yeast cells are obviously less complex than human neurons, basic metabolic pathways involved in neurodegeneration are well conserved in S. cerevisiae.

To be useful and significant, a yeast model of a particular disease must recapitulate the crucial events preceding cell death that manifest during the course of the human disorder. Two basic strategies are usually followed in the construction of yeast models of human diseases depending on genetic and pathophysiological constraints. In most cases, human disorders result from a loss of function of the disease gene-encoded protein. In these cases, when the human disease gene is conserved from yeast to human, functional complementation studies will determine whether the human disease gene product is able to partially or fully replace the function of the yeast gene product. If complementation occurs, human disease gene mutant alleles are expressed in yeast and tested for functionality as for mutations in the $\mathrm{Cu}-\mathrm{Zn}$ superoxide dismutase gene responsible for ALS (6). If complementation does not occur, the disease mutations, frequently involving conserved protein residues, are alternatively introduced in the yeast protein and subsequently analyzed as reported for mutations in the adenine nucleotide translocator $(A N T 1)$ responsible for cases of external progressive ophthalmoplegia (7).

In age-associated neurodegenerative disorders, such as PD or HD, the human disease genes are restricted to vertebrates. In these diseases, however, pathogenicity is believed to greatly result from a gain of function of the disease mutant protein. Mutant forms of the proteins huntingtin (htt) and $\alpha$-synuclein, responsible for HD and certain familiar forms of PD, respectively, undergo misfolding and damage several cellular structures, which leads to cell death. Yeast models of these disorders are constructed by expressing the human gene in yeast, providing paradigms in which the effect of the function gained by the disease gene mutant allele on the cellular physiology and metabolism can be conveniently studied. Here, we will focus on describing the construction of yeast models of this type of disorders.

A valuable yeast model of a gainof-function neurodegenerative disease based on the heterologous expression of a human gene is defined by several critical properties. The expression of the gene must duplicate essential cytotoxic events that appear during the course of the disease leading to a particular cellular phenotype that can be conveniently studied. Importantly, gene expression must be tightly regulated for both activation and levels of expression. Regulation is achieved by placing gene expression under the control of the appropriate promoters. Although models constructed with constitutive promoters (expression always on) are reported in the literature, their use is not recommended. In these models, upon yeast transformation to construct the yeast model, the expression of a toxic protein can result in either cell death prior to the formation of a yeast colony or in the 
generation of suppressor mutations in a subset of cells that make them competent to grow in the presence of toxic proteins, a situation that will adulterate the models. To achieve a regulated gene expression, researchers have used several natural or artificially modified inducible promoters that are turned either on or off upon the addition or subtraction of a particular inducer/repressor.

To date, most inducible yeast models of neurodegenerative diseases have been created by heterologous expression of human genes under the control of the strong GAL1 promoter, which is activated by galactose and repressed by glucose. Although these models have provided a significant amount of information, they generally suffer from a lack of regulation of expression level: expression is usually high, which makes toxicity far too acute as compared to the toxicity produced by the slow accumulation of mutant proteins observed in neurons from human patients. The expression of the toxic peptides can be so harmful for the cell that it can have a tendency to eliminate the plasmid expressing the mutated protein when episomal vectors are used. This problem can be solved by integrating the engineered gene into the yeast chromosomal DNA. We recognize that high expression of mutant proteins that produce acute toxic effects can save time and be effective when screening for drugs or genetic suppressors of cytotoxicity, but they are probably not the ideal system for analyzing metabolic or physiological disturbances that lead to cytotoxicity. Additionally, gene expression under the control of a galactose-inducible promoter introduces a metabolic constraint. $S$. cerevisiae is a facultative aerobe/anaerobe. It can produce energy via two different mechanisms (fermentation or respiration) depending on the available carbon sources and oxygen accessibility. In all reported models, expression is induced upon the transfer of cells to media containing $2 \%$ galactose, which is a fermentable substrate. The use of galactose-inducible promoters represents a significant disadvantage when the metabolic changes associated to switches in carbon source are relevant to the study. For example, the use of metabolism-independent inducers would allow for the study of cell toxicities under conditions where the cells are forced to exclusively respire, creating a better model of highly oxidative neuronal metabolism. In addition, the use of metabolicdependent inducers in the way mentioned above prevents studies in nondividing postmitotic cells using the yeast stationary phase model of aging (8). In this postmitotic state, energetic dependence on mitochondrial respiration and concomitant free radicals of oxygen (ROS) production highly resembles the situation in which neuronal cells age.

We have decided to create refined inducible yeast models of neurodegenerative disorders to increase their usefulness and relevance. We chose to begin by creating refined models of HD, a genetic disorder characterized by a selective neurodegeneration of the striatum and deep layers of the cerebral cortex (9). HD is caused by the expansion of a polyglutamine (polyQ) tract in htt. Here we have constructed yeast models of HD based on the expression of the polyQ domains of normal and pathological lengths under the control of different promoters, including the CUPI promoter, two different $\beta$-estradiol-inducible $G A L 1$ promoter systems, and the GAL1 promoter in a $\Delta$ gall mutant background. The different models are evaluated and compared.

\section{MATERIALS AND METHODS}

\section{Yeast Strains, Media, and Growth Conditions}

All experiments were performed with strains isogenic to the haploid reference strain W303-1A (MATa leu2-3,112 trp1-1 can1-100 ura3-1 ade2-1 his3-11,15). In the $\triangle$ gall mutant, the GALI gene was replaced with the kanMX deletion cassette.

Cells were cultured in either standard rich media YPRAF (2\% raffinose, $1 \%$ yeast extract, and $2 \%$ bactopeptone), YPD ( $2 \%$ dextrose, $1 \%$ yeast extract, and $2 \%$ bactopeptone), and YPG (2\% glycerol, $1 \%$ yeast extract, and $2 \%$ bactopeptone), or synthetic minimal medium $(0.67 \%$ yeast nitrogen base containing the required amino acids). Geneticin-resistant cells were grown on YPD plates containing 200 $\mathrm{mg} / \mathrm{L}$ geneticin.

Routinely, polyQ expression in yeast cells growing in YPRAF was induced for 6 and $24 \mathrm{~h}$ by supplementing the media with different concentrations of the appropriate inducers. Cells expressing polyQ constructs under the control of the $C U P I$ promoter were grown in the presence of 0 and $2 \mathrm{mM} \mathrm{CuSO}_{4}$. Cells expressing polyQ-GFP under the control of the $\beta$-estradiol-inducible $G A L 1$ promoter were grown in the presence of 0,10 , and $50 \mathrm{nM}$ $\beta$-estradiol (Tokyo Chemical Industry, Tokyo, Japan). Finally, cells expressing polyQ-GFP under the control of the GAL1 promoter were grown in the presence of $0 \%, 0.01 \%$, and $0.1 \%$ galactose.

\section{Plasmid Construction and Transformation}

As mentioned above, HD mutations result in an expansion of an N-terminal

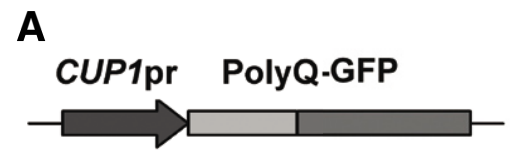

B

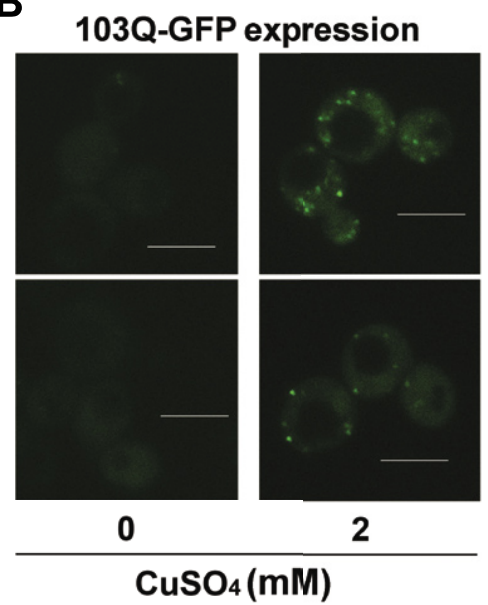

Figure 1. Copper-inducible model. (A) Representation of the polyQ-GFP expression cassette under the control of the $C U P 1$ promoter (CUP1pr). (B) Visualization of 103Q-GFP expression under the control of CUPlpr. Cells were grown for $6 \mathrm{~h}$ in YPRAF media in the presence or absence of $2 \mathrm{mM} \mathrm{CuSO}_{4}$. After induction, cells were mounted on slides and visualized under a fluorescence BX61 microscope, as described in the Materials and Methods section. The bar represents $5 \mu \mathrm{m}$.

polyQ domain in htt (encoded in the first exon of the gene) from 6-35 glutamines to more than 36 repeats. Mutant htt is cleaved both in vivo and in vitro to form $\mathrm{N}$-terminal fragments containing polyQ repeats. It is the general understanding that these fragments are actually the toxic forms of the protein. Although the exact identity of these cleavage fragments is not known, they appear to include approximately the first 150 residues of htt. For this reason, many laboratories, including ours, have modeled HD pathogenesis by the expression of exon 1 fragments, which cause toxicity and promote protein misfolding/aggregation in vivo and in cell models (reviewed in References 10 and 11). Two polyQ constructs (25Q and 103Q in the plasmid pYES2) previously described (12) were obtained from Dr. Michael Y. Sherman (Boston University, Boston, MA, USA). In brief, exon 1 sequences containing the first 17 amino acids followed by 25 or 103 glutamines were fused in frame to green fluorescent protein (GFP) at the $\mathrm{C}$ terminus of each construct, and a FLAG tag was attached to their $\mathrm{N}$ terminus (resulting in 25Q or 103Q). In each case, a KpnI/XbaI fragment containing the polyQ domain 


\section{Short Technical Reports}

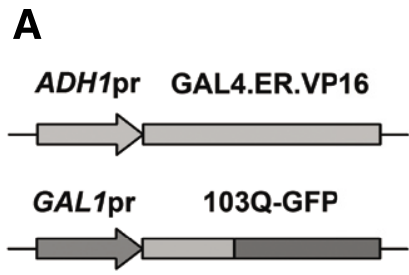

C

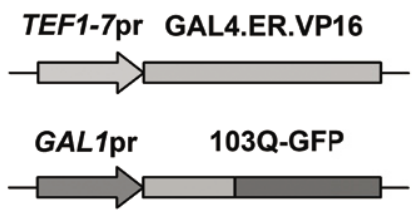

B

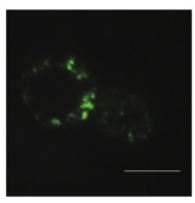

0

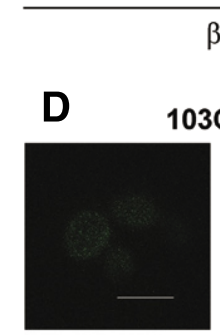

0
103Q-GFP expression

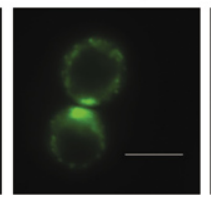

10

$\beta$-Estradiol(nM)

103Q-GFP expression

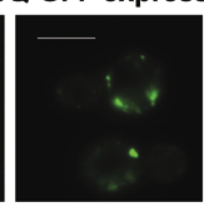

10

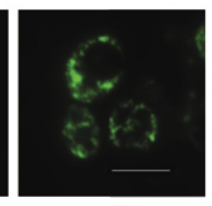

50

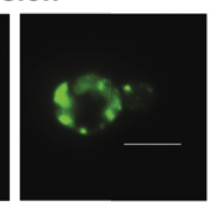

50

\section{$\beta$-Estradiol (nM)}

Figure 2. $\boldsymbol{\beta}$-Estradiol-inducible models. (A and C) Representations of the polyQ-GFP expression cassettes under the control of the $\beta$-estradiol-inducible promoter. Expression of the transactivator GLA4.ER.VP16 was placed under the control of either the $A D H I$ promoter ( $A D H I$ pr, in panel A) or TEF1-7 promoter (TEF1-7pr, in panel C) as explained in the text. (B and D) Visualization of polyQ-GFP expression under the control of the $\beta$-estradiol inducible promoters in systems using either $A D H 1 \mathrm{pr}$ (in panel B) or TEF 1-7pr (in panel D) to activate expression of the transactivator GLA4.ER.VP16. Cells were grown for $6 \mathrm{~h}$ in YPRAF media in the presence of 0,10 , and $50 \mathrm{nM}$ $\beta$-estradiol. Processing and visualization was performed as in Figure 1B. The bar represents $5 \mu \mathrm{m}$. fused to GFP was excised from these constructs and inserted into an integrative YIp351 plasmid (13) previously modified to express the proteins under the control of the GALI promoter (14). The polyQ constructs were also cloned in the plasmid YIplac128 as KpnI/XbaI downstream of the CUPI promoter (as HindIII/KpnI), obtained by PCR amplification using the W303-1A genomic DNA as the template. Additionally, the preparation of the $\beta$-estradiol-inducible GALI promoter systems required two independent constructs as explained below. In the first construct, the fusion cassette GAL4. ER.VP16 was expressed under the control of the alcohol dehydrogenase $1(A D H I)$ promoter in the plasmid pRS313 obtained from Dr. D. Picard (Dep de Biologie Cellulaire, Université de Genève, Genève, Switzerland). In the second construct, the GAL4.ER.VP16 fragment was cloned as NotI/SphI downstream a mutant form of the translation and elongation factor 1 (TEF 1) promoter (as EcoRI/NotI) in the plasmid YIplac204. The mutant promoter TEF $1-7$ retains $16 \%$ of $T E F 1$ activity (15) and was obtained as part of a collection

\section{microRNA biomarker validation just got easier}

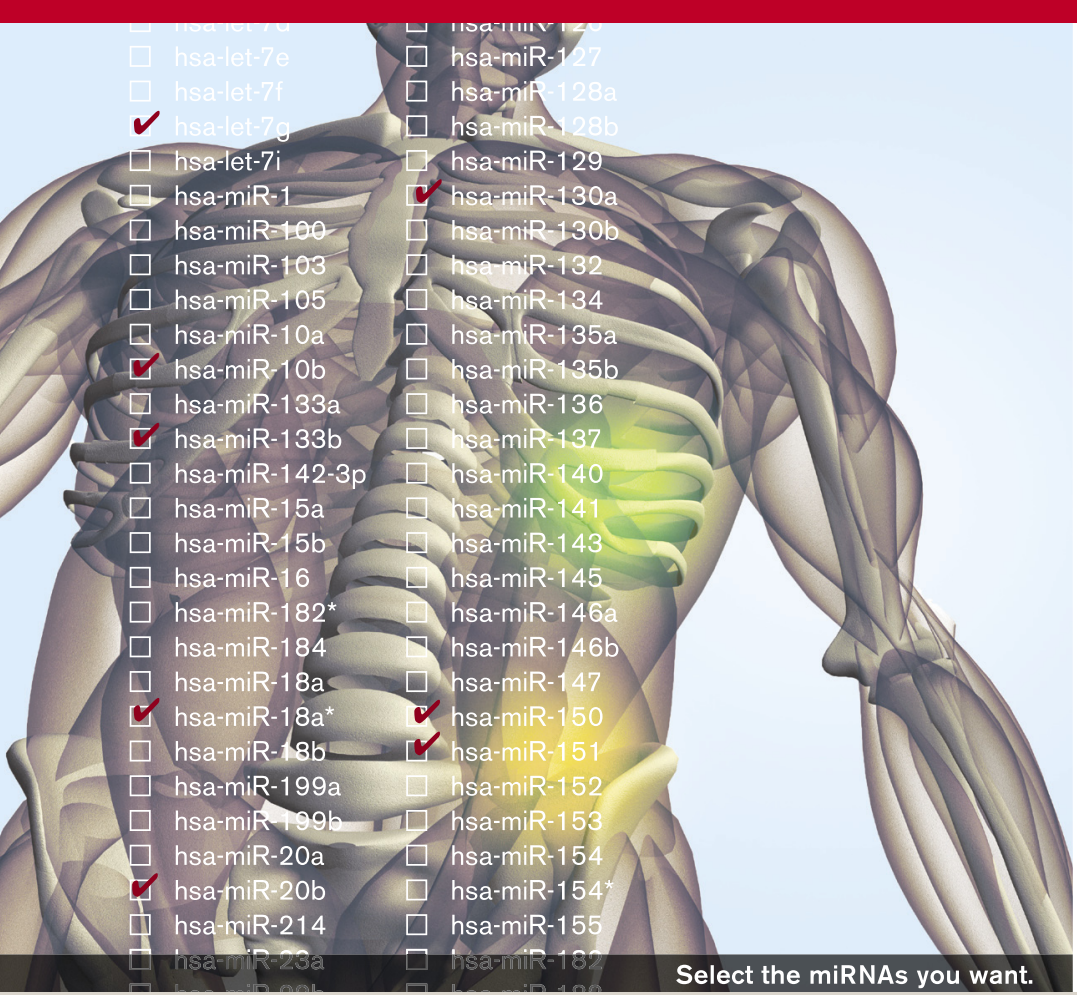

\section{FlexmiR Select Custom multiplex miRNA arrays}

- Accurate results within 4 hours

- Multiplex miRNA targets of your choice

- Quantitative data using standard curves

- Apply all the advantages of bead-based technology to your miRNA studies

Using Tm-normalized probes, FlexmiR liquid bead arrays provide high specificity in significantly less time than other miRNA assays.

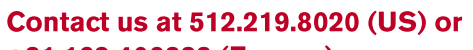
+31.162 .408333 (Europe)

Build your miRNA array with our online Custom Array Builder (CAB) at www.luminexcorp.com/CAB 
of TEF1-based promoter replacement cassettes from Dr. G. Stephanopoulos (Massachusetts Institute of Technology, Cambridge, MA, USA).

Standard procedures were used for the preparation and ligation of DNA fragments and for transformation and recovery of plasmid DNA from Escherichia coli strain DH5 $\alpha$ (16). Yeast were transformed by the method of Schiestl and Gietz (17). The one-step gene insertion method (18) was used to integrate linear plasmids at the LEU2 or TRP1 locus of yeast nuclear DNA of W3031A and W303 $\Delta$ gall strains. In the $\beta$-estradiol-inducible models, the plasmids containing the 103Q-GFP under the control of GALI promoter and the GAL4.ER.VP16 cassette were cotransformed into the same cells.

\section{Fluorescence Microscopy}

Wide-field fluorescence microscopy was performed to detect polyQ-GFP expression. We used an Olympus fluorescence BX61 microscope equipped with Nomarski differential interference contrast (DIC) optics (Olympus, Center Valley, PA, USA), a U-Plan-Apo $100 \times$ objective (NA 1.35), a Roper CoolsNAP HQ camera (Photometrics, Roper Scientific, Pleasanton, CA, USA), Lambda10-2 excitation and emission filter wheels (Sutter Instrument, Novato, CA, USA), and a $175 \mathrm{~W}$ Xenon remote source with liquid light guide. Images were acquired using SlideBook 4.01 (Intelligent Imaging Innovations, Denver, CO, USA).

\section{RESULTS AND DISCUSSION}

We tested the ability of four different promoters or expression systems to regulate gene expression in yeast models of neurodegenerative disorders by monitoring the expression of the first 17 amino acids (exon 1) of the human protein htt containing either 25Q or 103Q fused to GFP. GFP monitoring has allowed us to easily visualize protein expression, follow accumulation, and, for 103Q, macroaggregation. The fitness of the models was initially evaluated for the tightness of the system (the residual expression or leakage of the system when the inducer is not present) and its strength and regulation by using different concentrations of the inducer.

\section{Copper Inducible Models}

The CUPl gene codes for metallothionein, a protein that binds copper and mediates resistance to high concentrations of copper and cadmium (19). The $C U P 1$ promoter $(C U P I \mathrm{pr})$ is induced
A
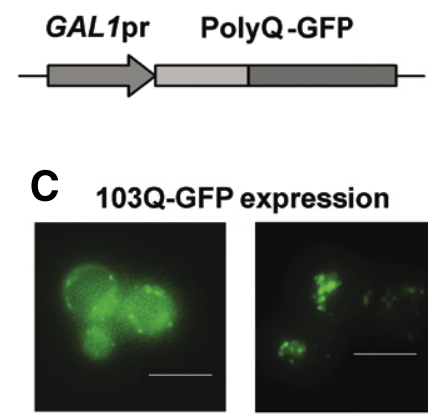

0.01

Galactose (\%)

0.1
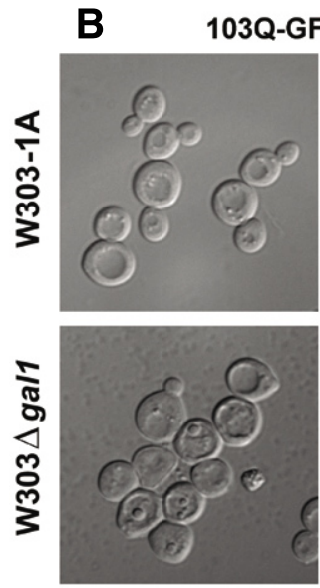

Nomarski
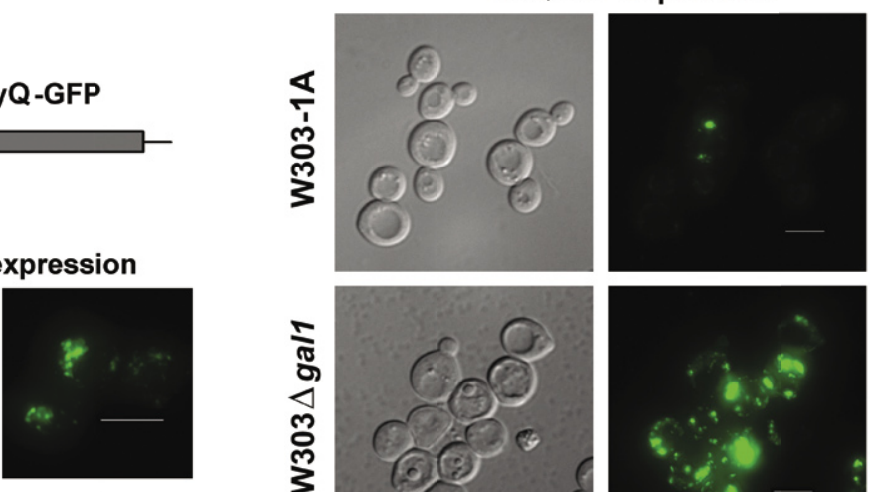

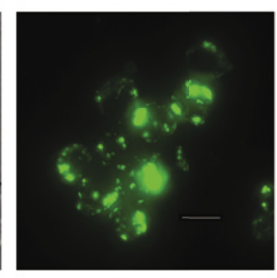

FITC

Figure 3. Gratuitous galactose-inducible model. (A) Representation of the polyQ-GFP expression cassette under the control of the GAL1 promoter. (B) Visualization of polyQ-GFP expression under the control of the GAL1 promoter. Cells were grown for $6 \mathrm{~h}$ in YPRAF media in the presence of $0.01 \%$ and $0.1 \%$ galactose. Processing and visualization was performed as in Figure 1B. (C) Comparison of the polyQ-GFP expression under the control of the GAL1 promoter in W303-1A and W303 $\Delta$ gall cells. Cells were grown for $24 \mathrm{~h}$ in YPRAF media in the presence of $0.1 \%$ galactose. Processing and visualization was performed as in Figure 1B. The bar represents $5 \mu \mathrm{m}$.

by the presence of copper in the growth media. The CUP1pr-polyQ-GFP constructs were integrated in single copy into the yeast $L E U 2$ locus (Figure 1A). Cells were grown in $2 \%$ YPRAF, and gene expression was induced with $0 \mathrm{mM}$ and $2 \mathrm{mM} \mathrm{CuSO}_{4}$. Although some interclone variability existed, we easily selected clones in which CUPlpr tightly regulated polyQ-GFP expression. Very low protein levels were expressed in the absence of copper $(0 \mathrm{mM})$ following growth for 6 $\mathrm{h}$ (Figure 1B) or $24 \mathrm{~h}$ (data not shown). By contrast, in the presence of $2 \mathrm{mM}$ $\mathrm{CuSO}_{4}$, protein expression was significantly increased after $6 \mathrm{~h}$ of induction (Figure 1B) and slowly continued at least up to $24 \mathrm{~h}$ (data not shown). While 25Q-GFP was completely diffused in the cytoplasm, 103Q-GFP was localized diffused in the cytoplasm and forming small aggregates as expected (Figure 1B). However, by using low copper concentrations, the amount of protein expressed was low, and the cells did not express significant toxicity after $24 \mathrm{~h}$ of induction (data not shown). Longer incubation times can result in the generation of spontaneous suppressor mutations that suppress potential mutant polyQ toxicities (Ocampo and Barrientos, unpublished observations). On the other hand, increasing the amount of inducer can produce deleterious effects given the high reactivity of copper, a redox transition metal able to activate oxygen through Fenton's reactions, thus producing free ROS (20). Copper should be used with caution, particularly when modeling diseases in which mitochondrial oxidative phosphorylation disturbances and ROS production could be involved in their pathogenic mechanisms, such as in the neurodegenerative disorders considered here (14).

\section{$\beta$-Estradiol Inducible Models}

Hormone ( $\beta$-estradiol) inducible systems for gene expression in yeast were devised by Dr. D. Picard (21). They are based on the constitutive expression of a transactivator fusion protein (GAL4. ER.VP16) formed by a GAL4 DNA binding domain, a $\beta$-estradiol receptor domain, and a virus protein 16 transcriptional activator, which can activate transcription of a gene placed under the control of a galactose inducible promoter $(G A L l \mathrm{pr})$. In the absence of the hormone, the fusion protein is repressed by the yeast chaperones from the heat shock protein 90 (Hsp90) family (21). Upon media supplementation with $\beta$-estradiol, the fusion protein is released from its repression, binds to $G A L 1 \mathrm{pr}$ through the GAL4 DNA binding domain, and the VP16 recruits the transcriptional machinery to start transcription.

Although $\beta$-estradiol inducible systems have been extensively used in biotechnological projects focused on protein production, they have not been used until now to generate yeast models of human diseases. Here we explored the potential application of these systems to create yeast models of neurodegenerative disorders. Our $\beta$-estradiol-inducible HD models 


\title{
Short Technical Reports
}

were created by transforming wild-type yeast cells with two constructs. In the first construct, polyQ-GFP was cloned downstream $G A L 1 \mathrm{pr}$ and integrated in a single copy into the yeast $L E U 2$ locus. The transformants were subsequently transformed with a second construct expressing GAL4.ER.VP16 under the control of either the strong constitutive $A D H 1$ promoter $(A D H I \mathrm{pr})$ (Figure $2 \mathrm{~A})$ or the weaker constitutive TEF $1-7$ promoter (TEF 1-7pr) (see Figure 2C) in a multicopy or integrative vector, respectively.

Cells were grown in $2 \%$ YPRAF media, and polyQ expression was induced by supplementing the media with 0,10 , and $50 \mathrm{nM} \beta$-estradiol. After $6 \mathrm{~h}$ of induction, gene expression was monitored by fluorescence microscopy. In the absence of $\beta$-estradiol, significant levels of expression were detected in cells containing the GAL4.ER.VP16 under the control of $A D H I \mathrm{pr}$ indicative of significant leakage of the system under these conditions (Figure 2B). Upon addition of 10 and $50 \mathrm{nM} \beta$-estradiol, polyQ expression was significantly higher, showing a good response to increasing concentrations of the inducer. Mutant polyQ was detected diffused in the cytoplasm as well as forming large macroaggregates indicative of high levels of expression after only 6 $\mathrm{h}$ of induction (Figure 2B). When GAL4. ER.VP16 expression was placed under the control of the weak TEF 1-7pr, the system was significantly tightly regulated, because no leakage was detected in the absence of $\beta$-estradiol after 6 or $24 \mathrm{~h}$ (Figure 2D). In the presence of 10 and $50 \mathrm{nM} \beta$-estradiol, a significant protein expression was observed. The increase was roughly proportional to the amount of hormone present in the media as visualized by the number, size, and fluorescence intensity of the polyQ aggregates detected inside the cells (Figure 2D).

However, we have observed that the GAL4.ER.VP16 construct alone produces some degree of toxicity, reducing the growth fitness of yeast cells (data not shown) presumably by trapping general transcription factors required at RNA polymerase II promoters (22). The VP16 toxic effect was significantly higher when its expression was under the control of the strong $A D H 1$ pr than lower, when its expression was under the control of the weak TEF 1-7pr. This toxic effect, which could not be completely eliminated even using very low $\beta$-estradiol concentrations, will interfere and potentially produce misleading interpretations when analyzing mutant polyQ-induced cytotoxicity. At this point, we cannot discard the fact that by using promoters even weaker than TEF 1-7pr, VP16 toxicity could be totally eliminated, a possibility that is currently being tested in our laboratory.

\section{Gratuitous Galactose-Inducible Promoters}

To generate a gratuitous galactoseinducible system, we have taken advantage of our originally reported $G A L 1$-promoterbased models (23) and safely mutate the galactose metabolic pathway. Gratuitous induction of the GAL promoters can be achieved by mutations of the chromosomal GAL1 gene (24). The GAL1 product, galactokinase, is the enzyme required for the first step in the galactose metabolism and catalyzes the conversion of galactose into galactose-1-phosphate. In a $\Delta$ gall strain, galactose metabolism is deactivated early in the pathway, avoiding accumulation of any potentially harmful

\section{We're Ready When You Are....}

\author{
Preclinical contract research in cancer \\ and infectious disease therapeutics
}

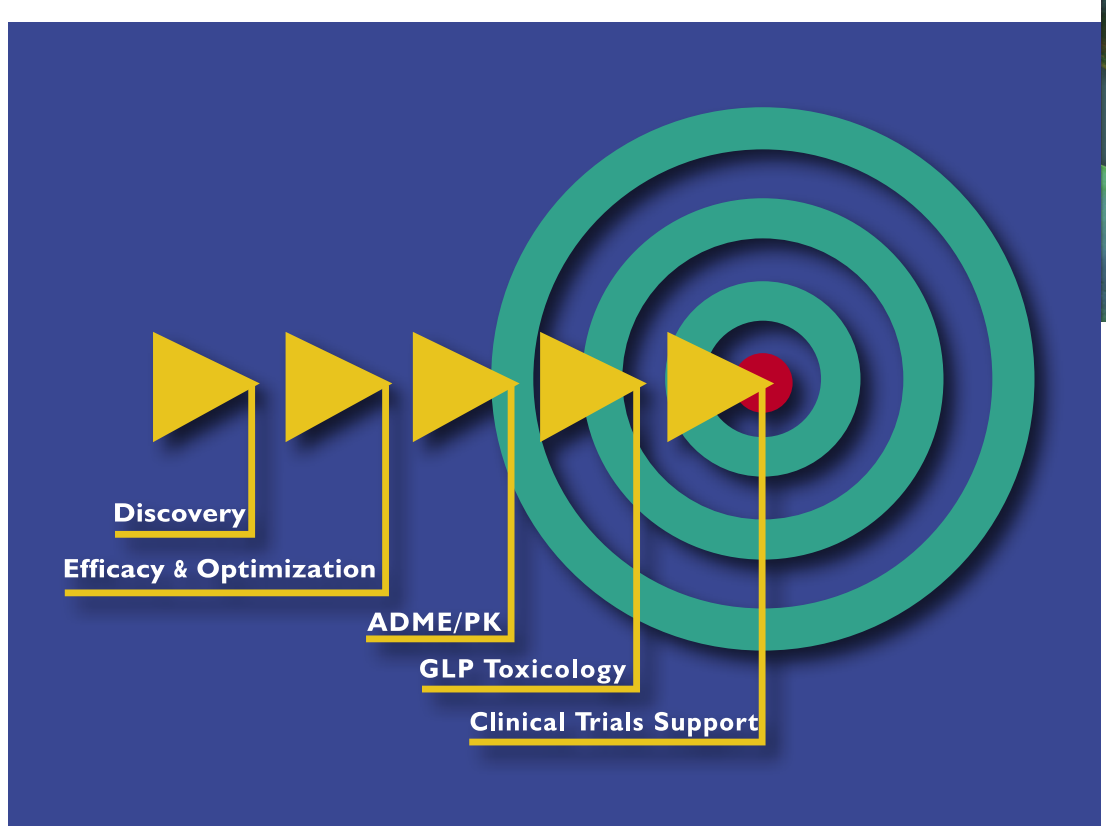

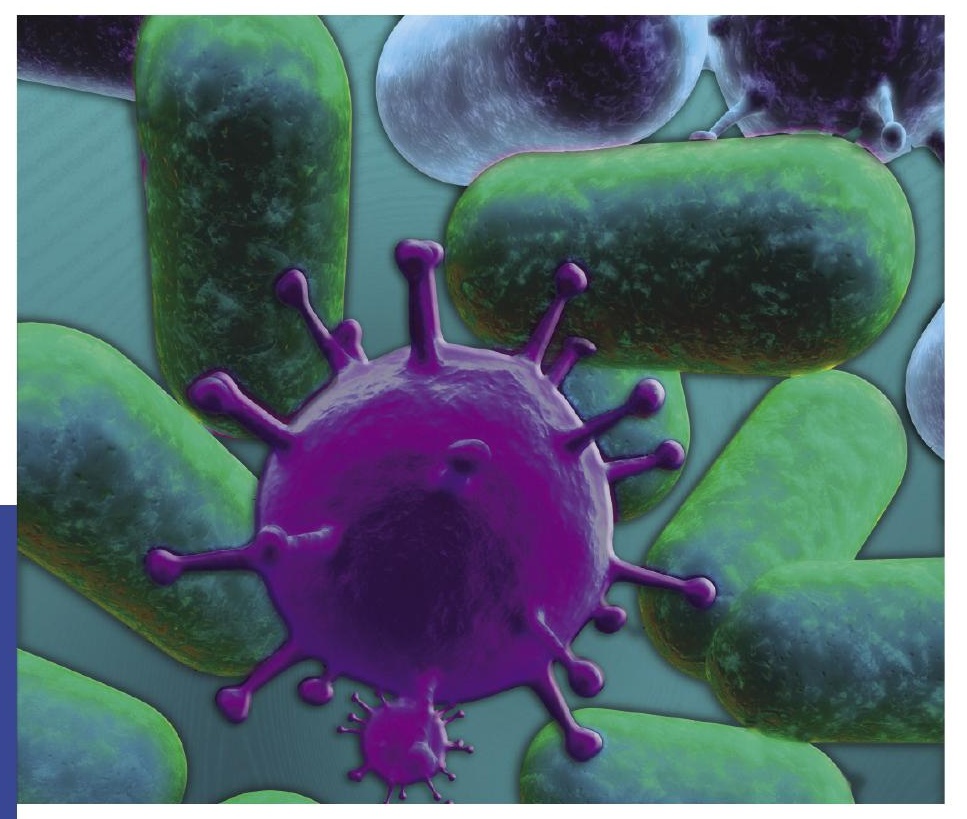

SOUTHERN RESEARCH

Legendary Discoveries. Leading Innovation.

Call us or visit our web site for more information.

www.SouthernResearch.org

(888) $322-1166$

001 (205) 581-2830

BusDev@SouthernResearch.org 
metabolite. Hence, galactose serves as a gratuitous inducer. To prepare these models, we have created a W303 $\Delta$ gall strain by replacing the full GALl gene with a kanamycin resistance cassette using standard molecular biology techniques. The W303 $\Delta$ gall strain failed to grow in media containing galactose but grew at a similar rate to the parental wild-type strain W303-1A in media containing fermentable (glucose or raffinose) or respiratory (ethanol-glycerol or lactate) substrates (data not shown). The W303 $\Delta$ gall strain was subsequently transformed with the integrative galactose-inducible expression plasmids YIp351-25Q-GFP and YIp351103Q-GFP to integrate the constructs into the chromosomal LEU2 locus.

Transformants were grown in $2 \%$ YPRAF (Figure 3B) for $8 \mathrm{~h}$ in the presence of $0.01 \%$ and $0.1 \%$ galactose. Protein expression was not detected in the absence of galactose (data not shown) and induced when galactose was added to the media even at concentrations as low as $0.01 \%$. In cells expressing 25Q-GFP, the protein was diffused in the cytoplasm, and no cytotoxicity was observed (data not shown). In cells expressing 103Q-GFP, the protein was diffused in the cytoplasm with a fraction that formed visible aggregates (Figure 3B) and produced cytotoxicity (data not shown). The levels of expression (Figure 3B) and 103Q-GFP toxicity (data not shown) correlated to the amount of galactose in the media. Thus, by adding $0.1 \%$ galactose, the levels of protein expressed as well as the number and size of the aggregates were further increased (Figure 3B).

Deletion of the gall gene prevents the metabolism of the galactose present in the media as explained above, thus maintaining constant levels of protein expression induced by small amounts of galactose. We compared 103Q-GFP expression regulated by the GALI inducible promoter in W303 $\Delta$ gall cells and W303-1A cells growing in either fermentable YPRAF (Figure 3C) or respiratory YPG media (Ocampo and Barrientos, unpublished data). After induction times shorter than $10 \mathrm{~h}$, polyQ protein cellular levels and homogeneity through the cell population were similar in both W303 gall and W303-1A cells (data not shown). However, after $24 \mathrm{~h}$ of induction with $0.1 \%$ galactose, protein

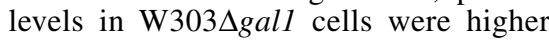
than in W303-1A cells and significantly homogenous in the whole cell population. For example, all W3034gall cells expressing the mutant 103Q-GFP protein contained large macroaggregates. In W303-1A cultures, the small amount of galactose present in the media was mostly metabolized before $24 \mathrm{~h}$ of incubation, resulting in variable levels of protein expression across the cell population with a significant proportion of cells in which the protein was not detected. In W303-1A cells expressing 103Q-GFP, the amount as well as the number and sizes of macroaggregates were significantly smaller (Figure 3C) than in W303 $\Delta$ gall mutant cells.

We conclude that induction of polyQ-GFP expression under the control of GALIpr in W303-1A cells by supplementing media containing fermentable $2 \%$ raffinose or respiratory $2 \%$ glycerol with small amounts of galactose is limited, but can be used for short-term studies. However, more versatile and solid models are obtained in the absence of galactose metabolism as explained above.

\section{Concluding Remarks}

All the yeast models of polyQ disorders presented here reproduced at least the basic hallmarks of neuronal degeneration observed in human patients represented by protein aggregation and cytotoxicity as described. However, not all of them can be used safely and cannot be equally recommended. The different expression systems tested to create the models have been evaluated considering their tightness, regulation of levels of expression, selftoxicity, and metabolic independence.

We would not recommend the use of models based on copper-induced polyQ expression given the strong possibility that the inducer will catalyze ROS production. The $\beta$-estradiol inducible models are strongly regulated and would hold promise if we were able to eliminate VP16 toxicity using very weak constitutive promoters for the expression of the GAL4.ER.VP16 transactivator.

Our current data suggest that the highly regulatable, nontoxic, gratuitous induction system based on GALI gene disruption to safely avoid galactose metabolization is the best choice for stable and metabolically independent polyQ expression in $S$. cerevisiae. In this system, cells can be grown in media containing either fermentable (i.e., raffinose) or respiratory (i.e., lactate or ethanol-glycerol) substrates; the polyQ expression, under the control of a Gal inducible promoter, is regulated by the addition of low concentrations of galactose to the media. The GALI disruption system allows for maintaining a stable galactose concentration throughout cell growth, thus resulting in permanent induction of galactose-inducible promoters. In addition, by using this system, nondividing yeast cells can also be induced to produce the recombinant protein of interest.

Finally, it is our hope that the data presented in this report for the yeast models of HD will be useful when modeling other diseases as well.

\section{ACKNOWLEDGMENTS}

We are supported by funds from the John T. Macdonald Foundation Center for Medical Genetics and the Miami Institute for Human Genomics at the University of Miami Miller School of Medicine. We thank Dr. Flavia Fontanesi and Darryl Horn for critically reading the manuscript.

\section{COMPETING INTERESTS STATEMENT}

The authors declare no competing interests.

\section{REFERENCES}

1. Samuel, D. 1996. Investigation of ancient Egyptian baking and brewing methods by correlative microscopy. Science 273:488490.

2. Botstein, D. 1991. Why yeast? Hosp. Pract. (Off. Ed.) 26:157-164

3. Barrientos, A. 2003. Yeast models of human mitochondrial diseases. IUBMB Life 55:8395.

4. Goffeau, A., B.G. Barrell, H. Bussey, R.W. Davis, B. Dujon, H. Feldmann, F. Galibert, J.D. Hoheisel, et al. 1996. Life with 6000 genes. Science 274:546-567.

5. Miller-Fleming, L., F. Giorgini, and T.F. Outeiro. 2008. Yeast as a model for studying human neurodegenerative disorders. Biotechnol. J. 3:325-338

6. Gunther, M.R., R. Vangilder, J. Fang, and D.S. Beattie. 2004. Expression of a familial amyotrophic lateral sclerosis-associated mutant human superoxide dismutase in yeast leads to decreased mitochondrial electron transport. Arch. Biochem. Biophys. 431:207214.

7. Fontanesi, F., L. Palmieri, P. Scarcia, T. Lodi, C. Donnini, A. Limongelli, V. Tiranti, M. Zeviani, et al. 2004. Mutations in $A A C 2$, equivalent to human adPEO-associated ANT1 mutations, lead to defective oxidative phosphorylation in Saccharomyces cerevisiae and affect mitochondrial DNA stability. Hum. Mol. Genet. 13:923-934.

8. Sinclair, D., K. Mills, and L. Guarente. 1998. Aging in Saccharomyces cerevisiae. Annu. Rev. Microbiol. 52:533-560.

9. Vonsattel, J.P., R.H. Myers, T.J. Stevens, R.J. Ferrante, E.D. Bird, and E.P. Richardson, Jr. 1985. Neuropathological classification of Huntington's disease. J. Neuropathol. Exp. Neurol. 44:559-577.

10. Landles, C. and G.P. Bates. 2004 Huntingtin and the molecular pathogenesis of Huntington s disease. EMBO Rep. 5:958 963.

11. Li, S.H. and X.J. Li. 2004. Huntingtin and its role in neuronal degeneration Neuroscientist 10:467-475.

12. Meriin, A.B., X. Zhang, X. He, G.P. Newnam, Y.O. Chernoff, and M.Y. Sherman. 2002. Huntington toxicity in yeast model depends on polyglutamine aggregation mediated by a prion-like protein Rnq1. J. Cell Biol. 157:997-1004.

13. Hill, J.E., A.M. Myers, T.J. Koerner, and A. Tzagoloff. 1986. Yeast/E. coli shuttle vectors with multiple unique restriction sites. Yeast 2:163-167. 


\section{Short Technical Reports}

14. Solans, A., A. Zambrano, M. Rodriguez, and A. Barrientos. 2006. Cytotoxicity of a mutant huntingtin fragment in yeast involves early alterations in mitochondrial OXPHOS complexes II and III. Hum. Mol. Genet. 15:3063-3081.

15. Nevoigt, E., J. Kohnke, C.R. Fischer, H. Alper, U. Stahl, and G. Stephanopoulos. 2006. Engineering of promoter replacement cassettes for fine-tuning of gene expression in Saccharomyces cerevisiae. Appl. Environ. Microbiol. 72:5266-5273.

16. Sambrook, J., E.F. Fritsch, and T. Maniatis. 1989. Molecular Cloning: A Laboratory Manual. CSH Laboratory Press, Cold Spring Harbor, NY.

17. Schiestl, R.H. and R.D. Gietz. 1989. High efficiency transformation of intact yeast cells using single stranded nucleic acids as a carrier. Curr. Genet. 16:339-346.

18. Rothstein, R.J. 1983. One-step gene disruption in yeast. Methods Enzymol. 101:202211.

19. Butt, T.R., E.J. Sternberg, J.A. Gorman, P. Clark, D. Hamer, M. Rosenberg, and S.T. Crooke. 1984. Copper metallothionein of yeast, structure of the gene, and regulation of expression. Proc. Natl. Acad. Sci. USA $81: 3332-3336$

20. Sutton, H.C. and C.C. Winterbourn. 1989 On the participation of higher oxidation states of iron and copper in Fenton reactions. Free Radic. Biol. Med. 6:53-60.

21. Louvion, J.F., B. Havaux-Copf, and D. Picard. 1993. Fusion of GAL4-VP16 to a steroid-binding domain provides a tool for gratuitous induction of galactose-responsive genes in yeast. Gene 131:129-134.

22. Berger, S.L., B. Pina, N. Silverman, G.A. Marcus, J. Agapite, J.L. Regier, S.J. Triezenberg, and L. Guarente. 1992. Genetic isolation of $A D A 2$ : a potential transcriptional adaptor required for function of certain acidic activation domains. Cell 70:251-265

23. Engelender, S., A.H. Sharp, V. Colomer, M.K. Tokito, A. Lanahan, P. Worley, E.L. Holzbaur, and C.A. Ross. 1997. Huntingtinassociated protein 1 (HAP1) interacts with the p150Glued subunit of dynactin. Hum. Mol. Genet. 6:2205-2212.

24. Hovland, P., J. Flick, M. Johnston, and R.A. Sclafani. 1989. Galactose as a gratuitous inducer of GAL gene expression in yeasts growing on glucose. Gene 83:57-64.

Address correspondence to Antoni Barrientos, Department of Neurology and Biochemistry \& Molecular Biology, The John T. Macdonald Center for Medical Genetics, Universtiy of Miami Miller
School of Medicine, 1600 NW 10th Ave., RMSB \#2067, Miami, FL 33136, USA. e-mail:abarrientos@med.miami.edu

To purchase reprints of this article, contact:Reprints@BioTechniques.com

\section{WEUJOSGIEISE 2008 Booth 314}

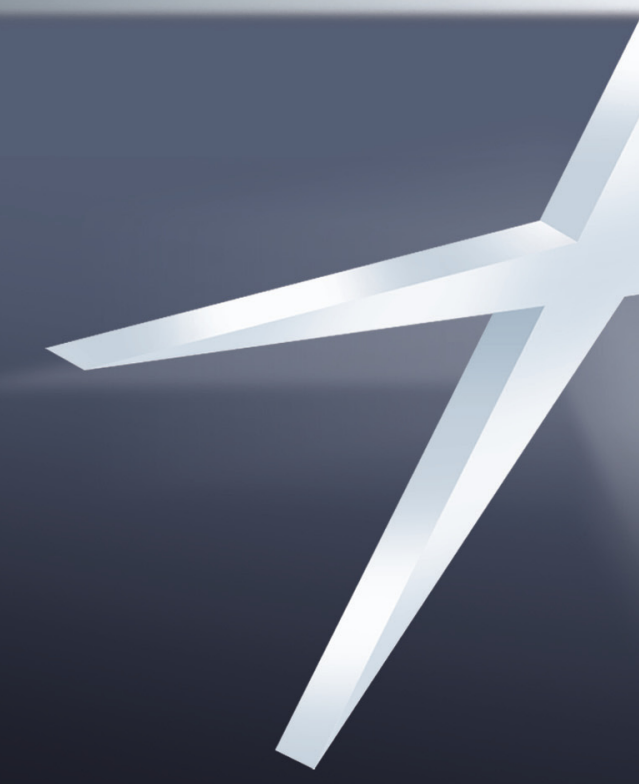

Contact ART• optix@art.ca • www.art.ca 514-832-0777 • 888-278-7888

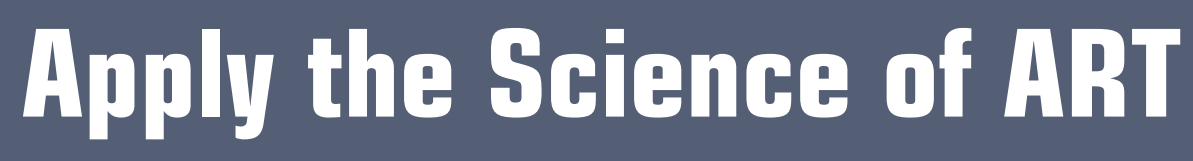

ONCOLOEY • CNS • CV • ADME/TOX • DRUE DISCOVERY ilighest Prouen Sensitjuity Highest Besolution Qluantitatiue Besults Jubutitu 3 5oftware Iptiog ot fusjum module

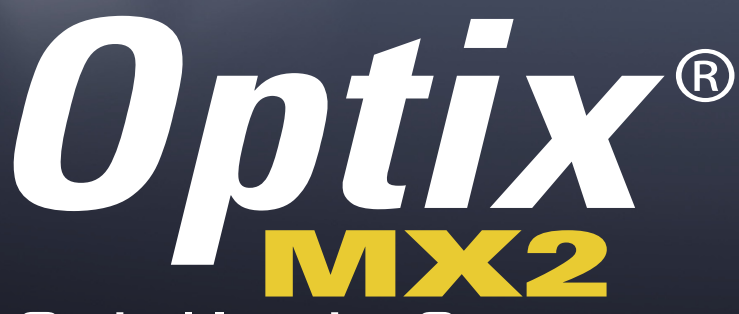

optioal Imaging System

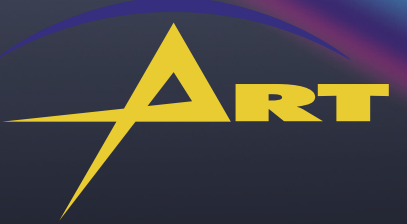

ART Advanced Research Technologies Inc. 